\title{
Effect of Dredging Rate, Sintering Time, Cooling Media on Corrosion Results of Carbon Steel Dredging
}

\author{
Dani Irawan ${ }^{a^{*}}$, Ahmad Dony Mutiara Bahtiara, Rahayu Mekar Bisono ${ }^{\mathrm{b}}$ \\ ${ }^{a}$ Mechanical Engineering, State Polytechnic of Malang Campus Kediri, Kediri Indonesia \\ ${ }^{b}$ Mechanical Engineeiring, Ralilway System Polytechinic of Madirun, Madiun, Indonesia
}

\begin{abstract}
A B S T R A C T
Metal reinforcement that has an impact on improving mechanical properties can occur in various ways, including by strain hardening mechanisms, solid solutions, second phase, prespitations, disperse, grain refinement and texture. In recent years, another method has been developed to obtain good mechanical properties without adding alloy elements, namely by the method of comprehensive plastic deformation (Severe Plastic Deformation). The purpose of this research is to conduct a study of rolling using medium carbon steel with a treatment temperature above recrystallization, rolling rate, sintering time, cooling media as an effort to develop and design new innovations about steel structures being rolled. The study of developing rolling techniques that can be applied in industry and can be applied as learning material / media in higher education requires structured research stages. The best impact value from this temper process is a temperature of $550{ }^{\circ} \mathrm{C}$ for 1 hour where the impact value is $1.58 \mathrm{~J} / \mathrm{mm}^{2}$ with air conditioning media. In the rolling process above the recrystallization temperature $800{ }^{\circ} \mathrm{C}$ deformation $20 \%$ the impact value is $1.7 \mathrm{~J} / \mathrm{mm}^{2}$ where the toughness indicates a ductile fracture while the deformation is $10 \%$ the impact value
\end{abstract}

Keywords: Cooling Media, Corrosion Results, Dredging Dredging rate, Sintering Time.

Article History

Received 08 December 19

Received in revised form 07 February 20

Accepted 26 July 21

\section{Introduction}

The development of material technology and microtechnology engineering has driven enormous changes in the use of materials, especially steel in the industrial world. Along with the development, steel is needed with the characteristics and characteristics that are appropriate to the conditions when applied [1]. Therefore we need steel with strong characteristics, tough and corrosion resistant [2]. The toughness which is still low on a steel will cause easy breakage and easy corrosion. In recent years, another method has been developed to obtain good mechanical properties without adding alloy elements, namely by the method thorough plastic deformation (Severe Plastic Devormation). The overall deformation process is the process of forming metals in which the plastic strain given to the metal or material is very large so that it produces fine grain (Ultra Fine Grain). A research, [3] obeseved about Intermittent drying of paddy rice is fully investigated both theoretically and experimentally. A model was developed to represent simultaneous heat and mass transfer for drying stages and mass transfer for tempering one. And both specimen was cylindrical and spherical coordinates assuming non-constant paddy rice physical properties. Further, a set of experiments are designed and carried out in a lab-scale fluidized bed dryer to estimate the moisture diffusivity of rice and to evaluate the effects of different parameters. The model estimates show good agreement with experimental data. Cylindrical model slightly better fits the experimental results specially after tempering stage. Simulation results reveal that thermal equilibrium is rapidly reached within the first two minutes. Air velocity shows to have no significant effect on drying rate when fluidization condition is prevailed. In addition, drying rate is drastically improved after applying tempering stages as a result of moisture gradient removal.

The deformation process is done, among others, can be done by way of the hot working or cold. The deformation process by way of rolling give effect to the violence prevalent in steel. According to [4] rolling process above the recrystallization temperature from the results of the hardness and toughness test at a temperature of $800^{\circ} \mathrm{C}$ deformation of $20 \%$ its value above the raw material (raw material), namely a hardness value of $420 \mathrm{BHN}$ and a toughness of $1.7 \mathrm{~J} / \mathrm{mm}^{2}$ so that it can be concluded that the effect of the rolling process is above the recrystallization temperature at a temperature of $800{ }^{\circ} \mathrm{C}$ deformation $20 \%$ better than the initial material (raw material). The research explained that the rolling process and the temperature above the crystallization temperature had an increase in the hardness characteristics of the metal because the structure of the metal grains was getting smaller. But on the other hand the advantages of rolling process results have their own weaknesses against corrosion resistance,

* Corresponding author. Phone : +62-857-355-42375.

E-mail address: the_boymaster2@yahoo.com. 
such as the results of research conducted by explaining that the corrosion rate of rolling results against $\mathrm{NaCl}$ decreases with increasing deformation but it cannot be concluded that grain size affects the rate corrosion due to polarization testing only measures the rate of surface corrosion. From the results of these studies indicate that increasing deformation and structural changes of steel experience a low corrosion rate. [5] research results of the rolling process above the recrystallization temperature from the results of the hardness and toughness test at a temperature of $800{ }^{\circ} \mathrm{C}$ deformation of $20 \%$ value above the raw material (raw material), namely a hardness value of $420 \mathrm{BHN}$ and a toughness of $1,7 \mathrm{~J} / \mathrm{mm}^{2}$ so that it can be concluded that the effect of the rolling process above the recrystallization temperature at a temperature of $800{ }^{\circ} \mathrm{C}$ deformation is $20 \%$ better than the initial material (raw material) [6]. The rate of corrosion of $\mathrm{NaCl}$ decreases with increasing deformation but it cannot be concluded that grain size affects the rate of corrosion because polarization testing only measures surface corrosion rates. A reaseach observed about grain boundary on Fe-Mn-Al$\mathrm{C}$-Cr annealed steel at $1100{ }^{\circ} \mathrm{C}$ for $30 \mathrm{~min}$ after hot rolling with $66 \%$ and $80 \%$ reduction. The fractions of low- $\Sigma$ coincidence site lattice (CSL) boundaries have exceeded $60 \%$ after hot rolling, and reached $77 \%$ after annealing treatment. Whether after hot rolling or annealing, the fractions of $\Sigma 9$ and $\Sigma 27$ in samples with $80 \%$ reduction were obviously higher than those with $66 \%$ reduction. It indicated that hot rolling reduction was the key to realize grain boundary character distribution optimization [7] [8], [9]. On this research the effect of rolling treatment under recrystallization temperature that has been done, after the optimal values are taken, the results obtained are still below the raw material (raw material), so it can be concluded that the effect of rolling under recrystallization temperature is only decrease its mechanical properties. According to this reserch tempering can reduce the value of violence and tensile strength. [10] The results of hardness testing on $8 \mathrm{~mm}$ thick steel plates show the smaller the diameter of the rolling the higher the price of hardness, the highest hardness price is in the tensile radius of $1000 \mathrm{~mm}$ that is equal to $225.5 \mathrm{kglmm}^{2}$, and the lowest hardness price is in the area tensile material of $208.4 \mathrm{~kg} / \mathrm{mm}^{2}$. Explanation from some of the above studies, the researchers concluded with a deformation level, and crystallization temperature produces a low corrosion rate[11]. So the researchers took the existing problems to do further research with the aim to produce strength that is tough and high corrosion rate. To produce in accordance with the objectives of the study, researchers conducted tests with sintering time and air conditioning media [2].

The purpose of this research is to conduct a steel rolling study medium carbon with a treatment temperature above recrystallization, rolling rate, sintering time, cooling medium as an effort to develop and design new innovations about steel structures being rolled [12]. The purpose of this research is to conduct a study of rolling using medium carbon steel with a treatment temperature above recrystallization, rolling rate, sintering time, cooling media as an effort to develop and design new innovations about steel structures being rolled undergoing the rolling process with treatment of rolling rate, sintering time and air conditioning media [13]. The purpose of the urgency of the research is that the pursuit of this research is carried out because of the urgent need to find a solution to the problem is that steel is widely used in various needs in the development of technology in various fields [14]. By far the most visible requirement is the need for manufacturing technology such as: manufacturing vehicle equipment, etc. So that the steel users have the results of rolling hoops have characteristics that match the needs such as resistance to corrosion, the level of hardness [15]. The target to be achieved is to find new breakthroughs by utilizing the characteristics contained in steel with a variety of treatments to get maximum results. One of the heat treatment processes in steel is hardening, which is the process of heating the steel to temperatures in the area or above the critical area followed by rapid cooling called quench [16]. The second is the method of comprehensive plastic deformation (Severe Plastic Deformation). The overall plastic deformation process is the process of forming metals in which plastic strain which is given to the metal or material being processed is very large so as to produce a smooth grain (ultra fine grain) [17]. The deformation process is done, among others, can be done by way of the hot working or cold. With some of the above treatment for cooling media using air whose main purpose is to produce deformed steel to resist corrosion [18].

\subsection{Bumping}

Scraping is the process of flexing the material using plastic properties [19]. The main purpose of the flexing process is to form a plate or the formation of a workpiece with the desired diameter / radius of a certain curvature. To produce a cylindrical or conical construction (for example a boiler, tank, pipe), or structure, a round plate bending machine is needed [20]. This machine consists of three rollers with the same diameter. Two of them are fixed and the other one can be set. Metal plates come in between the three rollers, and flexing occurs. The final diameter can be adjusted by adjusting the third roller: the closer it is to the fixed roller, the smaller the final diameter, the bending of the plate can be in the shape of a circular cone round in the gap between them. Movement on a small flexing machine is done by hand, whereas it is large with a motor[21], [22].

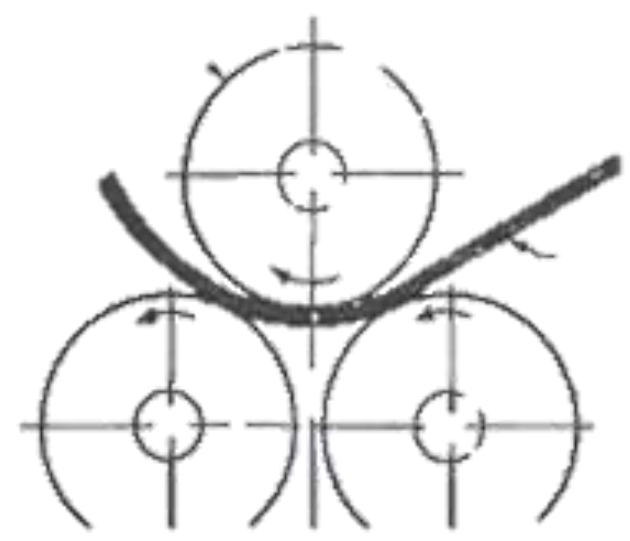

Figure 1. Caption Centered 


\subsection{Mechanical Properties of Steel}

Some important mechanical properties, among others (Zainuri, 2008: 102):

1. Strength (Strength)

State the material's ability to accept stress without causing the material to break. This strength is of several kinds depending on the type of load at work, namely tensile strength, compressive strength, shear strength, torsional strength and flexural strength.

2. Hardeness

It can be defined as the ability of a material to withstand scratches, abrasion (abrasion), and identification (penetration). This property is related to wear resistance. Violence also has a correlation with strength.

3. Ductility

State the ability of the material to accept stress without causing permanent deformation after the threshold is removed. The elasticity also states how much elastic deformation can occur before permanent deformation begins, in other words resilience states the ability of the material to return to its original shape and size after receiving a load that causes deformation.

4. Stiffness

Stating the ability of materials to accept stress or load without causing deformation or deflection.

\subsection{Deformation Process}

The effect of temperature on the processes of formation is change material properties and behavior. In general, an increase in temperature will result in a decrease in the strength of the material, an increase in ductility and a decrease in the rate of strain hardening in which the change results in material ease of deformation. Based on the temperature of the material at the time of this deformation, the metal forming process can be classified into two major groups, namely : Hot working, and Cold working[23].

\subsection{Sintering Process}

Sintering is a heating process below the melting point in order form a new crystalline phase as desired and aim to help react the constituent materials both ceramic and metal materials [24]. The sintering process will have a considerable effect on the formation of the crystalline phase of the material. The phase fraction formed generally depends on the sintering time and or temperature. The greater the sintering temperature it is possible the faster the crystal formation process [25]. The size of the temperature also affects the shape and size of the gap and also affects the structure of crystal growth[26]. Sintering temperatures can be determined from thermal experiments such as DTA, DTG, and DSC. Based on the results of this experiment obtained melt temperature other than decomposition temperature. Each composition of certain compounds has a melting point. Sintering of ceramic materials is usually determined around $75 \%$ of the total melting point [27]. In the sintering process, a new phase formation process takes place through a heating process where during the reaction the forming component is still in the solid form of the powder mixture. It is intended that the grains (grains) in adjacent particles can react and bind. The solid phase sintering process is divided into three solids, namely:
1. Early stage

At this initial stage atomic bonds are formed. Contact between particles forms a neck that grows into grain boundaries between particles. Growth will become faster with an increase in sintering temperature. At this stage shrinkage also occurs due to smooth porosity surfaces.

2. Intermediate stage

At this stage, particle desification and growth occur, ie small grains dissolve and join large grains. Accommodation of this grain shape results in compaction the better one. At this stage the porosity removal is also carried out. As a result of the grain boundary shift, porosity begins to interconnect and form a cylinder on the side of the grain.

3. The final stage

The phenomenon of desification and grain growth continues at a slower rate than before. Likewise with the porosity removal process, grain boundary shifts continue. If the grain boundary shift is slower than porosity, the porosity will appear on the surface and are interconnected. However, if the grain boundary shift is faster than porosity, the porosity will precipitate in the product and will be difficult to remove. The resulting product is expected to have a high density and homogeneous, then the homogenization process must occur in the sintering process. If there is an oxide layer on the metal powder, the expected sintering process can be slower. In addition to this oxide layer, the resulting product becomes more brittle, the oxide layer also inhibits the diffusion process between powder particles during sintering and increases the sintering temperature. The oxide layer attached to the powder is formed due to contact between the surface of the powder with air and due to the treatment received by the powder during the powder metallurgical production process. Oxides in powders can be minimized by flowing reduction gases before or during sintering.

\subsection{Observation of Micro Structure}

Microstructure observation aims to determine changes in microstructure from the material both before and after undergoing heat treatment. The structure, hardness and ongoing transformation of the HAZ (Heat Affected Zone) area can be read immediately on a diagram of a continuous cooling transformation or a CCT (Continuous Colling Transformation) diagram When the steel has cooled to the point "a" $( \pm 6800 \mathrm{C})$, the ferrite will begin to be deposited from austenite. This transformation continues and only ends when the point "b" $( \pm 5900 \mathrm{C})$ is reached and then replaced by the pearlit deposition transformation which will end at point "c" $( \pm 5200 \mathrm{C})$. From the discussion above it can be analyzed that after cooling the structure formed is ferrite and pearlite[28].

Usually a continuous cooling transformation diagram shows also the hardness that steel will have after it cools following a certain thermal cycle. Measuring the cooling time from $8000 \mathrm{C}$ to $5000 \mathrm{C}$ and combining with the CCT diagram of the same steel, the structure and hardness of the steel in the HAZ area can be determined. The diagram of the continuous cooling transformation can change due to changes in the maximum temperature that occurs[29]. Generally when the maximum temperature rises, curves that indicate the occurrence of certain structures 
in the diagram move to the right that lead to the stabilization of martensite formation, if this happens it is clear that the heat treatment results become harder[30].

A research observed about the tensile and fatigue tests were conducted on precipitate strengthened $\mathrm{Cu}-\mathrm{Ni}-\mathrm{Si}$ alloy. The fatigue properties of specimens with cold working and without cold working in air, as well as that of the specimen with cold working in salt atmosphere were investigated. The results show that the monotonic tensile strength and the yield strength are improved obviously, while the elongation decreases by cold working. All the fatigue cracks initiate from the specimen surface by slip deformation. In air condition, the specimens with cold working show shear mode fracture with transgranular crack propagation and it is caused by the obvious texture, while that without cold working show normal mode fracture with both transgranular and intergranular crack propagation. However, in salt atmosphere condition, the specimens with cold working show normal mode fracture with totally intergranular crack propagation[30].

\section{Methods}

The research method used is an experimental method. Samples to be treated are selected according to materials that are widely used in industry. The material used in this study is medium carbon steel. The material is then heated to a recrystallization temperature by rolling. The specimen consisted of 5 specimens with a thickness of $4 \mathrm{~cm}$ with a length of $15 \mathrm{~cm}$. the workpiece is heated with a temperature of recrystallization with a temperature of $700 \mathrm{C}$, then cooled with free air and thickness of the deformation of $5 \%, 10 \%, 15 \%, 20 \%, 25 \%$. In addition, raw materials which were not treated were prepared to compare the results before treatment and after rolling. After that, the micro structure test, the level of corrosivity, are carried out. The specimen will pass the microstructure test, hardness test and tensile test. The results of this study are expected to be used as a guide in the rolling process in order to produce maximum violence.

The equipment used during this research are:

- Furnace Heating ( Furnace Naber)

- Thermocouple Type-K

- Hardener

- Calibration term

- Clamp specimen

- Polisher Optical microscope

- VB Microscope

- Brinell hardness test equipment

- Machine Scrap

- Torsee Type AMU10 tensile testing machine

The materials used in this study are as follows:

- Medium carbon steel which is a material used as a car rear spring (leaf spring) which is applied to the palm harvester's blade.

- Resin and hardener.

- Sand paper with grades 120, 240, 400, 600, 800, 1000, 1200 and 1500.

- Digital etching solution $5 \%$
- Panel Fabric

\section{Result}

Based on the results of hardness testing that the hardness with the most optimal value occurs at a temperature of $7000 \mathrm{C}$ with a deformation rate of $20 \%$ which is 405 on the BHN scale, followed at $550^{\circ} \mathrm{C}$ for 1.5 hours with water cooling media which is $333 \mathrm{BHN}$ and at a temperature of $550^{\circ} \mathrm{C}$ for 1.5 hours with ice water cooling media, and then up to the lowest hardness value of $288.6 \mathrm{BHN}$ at $600{ }^{\circ} \mathrm{C}$ with $5 \%$ deformation. Based on the results of the toughness test, it can be seen that toughness with the most optimal value occurs at $605 \mathrm{C}$ with the deformation rate of $5 \%$ is $2.36 \mathrm{~J} / \mathrm{mm}^{2}$, followed by deformation at a temperature of $655^{\circ} \mathrm{C}$ with a deformation rate of $10 \%$ which is equal to $2,4 \mathrm{~J} / \mathrm{mm}^{2}$ and subsequently to the lowest toughness value of $1.3 \mathrm{~J} / \mathrm{mm}^{2}$ at $750{ }^{\circ} \mathrm{C}$ with $20 \%$ deformation.

\section{Conclusion}

The conclusions that can be drawn from this study are:

The toughness and shape of the medium carbon steel fracture obtained from the impact test results, namely:

- In the process of forging $550^{\circ} \mathrm{C}$ for 1 hour the impact value is 1,39 $\mathrm{J} / \mathrm{mm}^{2}$ and for 2 hours the impact value is $1.45 \mathrm{~J} / \mathrm{mm}^{2}$ with ice water cooling media whose toughness shows brittle fracture, while the temperature of $550^{\circ} \mathrm{C}$ during 1 hour the impact value is $1.58 \mathrm{~J} / \mathrm{mm}^{2}$ with the air conditioning medium whose toughness shows a ductile fracture. The best impact value from this temper process is a temperature of $550^{\circ} \mathrm{C}$ for 1 hour where the impact value is $1.58 \mathrm{~J} /$ $\mathrm{mm}^{2}$ with air conditioning media.

- In the process of rolling under recrystallization temperature $650{ }^{\circ} \mathrm{C}$ deformation $10 \%$ the impact value is $2.13 \mathrm{~J} / \mathrm{mm}^{2}$ and $5 \%$ deformation the impact value is $2.3 \mathrm{~J} / \mathrm{mm}^{2}$ while the temperature 600 ${ }^{\circ} \mathrm{C}$ deformation $5 \%$ the impact value is $2,37 \mathrm{~J} / \mathrm{mm}^{2}$ which shows that all toughness is a ductile fracture. The best impact value of the rolling process under this recrystallization temperature is $600{ }^{\circ} \mathrm{C} 5 \%$ deformation where the impact value is $2.37 \mathrm{~J} / \mathrm{mm}^{2}$.

- In the rolling process above the recrystallization temperature $800^{\circ} \mathrm{C}$ deformation $20 \%$ the impact value is $1.7 \mathrm{~J} / \mathrm{mm}^{2}$ where the toughness indicates a ductile fracture while the deformation is $10 \%$ the impact value.

\section{REFERENCES}

[1] R. M. Bisono and R. M. Yusron, "Optimization Multi Response on Electrical Discharge Machining Sinking Process Using Taguchi-Grey-Fuzzy Methods," Int. J. Sci. Eng. Inf. Technol., vol. 4, no. 2, pp. 209-214, 2020, doi: 10.21107/ijseit.v4i2.6705.

[2] J.-R. Zhao, F.-Y. Hung, and B.-J. Chen, "Effects of heat treatment on a novel continuous casting direct rolling 6056 aluminum alloy: cold rolling characteristics and tensile fracture 
properties," J. Mater. Res. Technol., vol. 11, pp. 535-547, 2021, doi: https://doi.org/10.1016/j.jmrt.2021.01.037.

W. J. Cantwell and J. Morton, "The impact resistance of composite materials - a review," Composites, vol. 22, no. 5, pp. 347-362, 1991, doi: https://doi.org/10.1016/00104361(91)90549-V.

[4] M. Assar, M. Golmohammadi, and S. J. Hashemi, "HEAT AND MASS TRANSFER CHARACTERISTICS OF

INTERMITTENT PADDY RICE DRYING :

EXPERIMENTAL AND THEORETICAL STUDY HEAT AND MASS TRANSFER CHARACTERISTICS OF INTERMITTENT PADDY RICE DRYING : EXPERIMENTAL AND THEORETICAL STUDY," no. December, 2012.

T. Shiwei et al., "Oxidation resistance of TiAl alloy improved by hot-pack rolling and cyclic heat treatment," Mater. Charact., vol. 178, p. 111196, 2021, doi:

https://doi.org/10.1016/j.matchar.2021.111196.

[6] C. Veera, B. A. Pradeep, C. Boopathi, R. K. Sanjeev, and V. Meganathan, "Comparison of geometrical accuracy and surface finish of cam profile generated by wire-EDM and CNC milling machine Comparison of geometrical accuracy and surface finish of cam profile generated by wire-EDM and CNC milling machine," pp. 0-8, 2020, doi: 10.1088/1757899X/764/1/012041.

[7] J. Liu, H. Wu, S. Yang, X. Yu, and C. Ding, "Effect of hot rolling reduction and annealing treatment on grain boundary character distribution in austenitic low-density Fe-Mn-Al-C-Cr steel," Mater. Lett., vol. 285, p. 128999, 2021, doi: https://doi.org/10.1016/j.matlet.2020.128999.

[8] L. Liang, S. Chen, and P. Li, "The evaluation of vehicle interior impact noise inducing by speed bumps based on multi-features combination and support vector machine," Appl. Acoust., vol. 163, p. 107212, 2020, doi: https://doi.org/10.1016/j.apacoust.2020.107212.

[9] R. K. Jha and P. D. Swami, "Fault diagnosis and severity analysis of rolling bearings using vibration image texture enhancement and multiclass support vector machines," Appl. Acoust., vol. 182, p. 108243, 2021, doi: https://doi.org/10.1016/j.apacoust.2021.108243.

[10] L. Chai et al., "A strategy to introduce gradient equiaxed grains into $\mathrm{Zr}$ sheet by combining laser surface treatment, rolling and annealing," Scr. Mater., vol. 196, p. 113761, 2021, doi: https://doi.org/10.1016/j.scriptamat.2021.113761.

[11] T.-A. Pan, Y.-C. Tzeng, H.-Y. Bor, K.-H. Liu, and S.-L. Lee, "Effects of the coherency of $\mathrm{Al} 3 \mathrm{Zr}$ on the microstructures and quench sensitivity of Al-Zn-Mg-Cu alloys," Mater. Today Commun., vol. 28, p. 102611, 2021, doi: https://doi.org/10.1016/j.mtcomm.2021.102611.

[12] I. P. Starodubtseva, D. V Kuznetsov, and A. N. Pavlenko, "Experiments and modeling on cryogenic quenching enhancement by the structured capillary-porous coatings of surface,” Int. J. Heat Mass Transf., vol. 176, p. 121388, 2021, doi: https://doi.org/10.1016/j.ijheatmasstransfer.2021.121388.

[13] T. Wang, Y. Hu, P. Fu, and H. Liu, "Quench detection method for superconducting magnets with a phase difference measurement system based on multiple-correlation," Fusion Eng. Des., vol. 170, p. 112658, 2021, doi: https://doi.org/10.1016/j.fusengdes.2021.112658.

[14] P. Hurley and J. P. Duarte, "Implementation of fiber optic temperature sensors in quenching heat transfer analysis," Appl. Therm. Eng., vol. 195, p. 117257, 2021, doi: https://doi.org/10.1016/j.applthermaleng.2021.117257.

[15] R. M. Yusron, R. M. Bisono, and M. Pramudia, "Effect Electrolyte Temperature and Electrode Distance to Electroplating Hard-Chrome on Medium-Carbon Steel," in Journal of Physics: Conference Series, 2020, vol. 1569, no. 4, doi: 10.1088/1742-6596/1569/4/042007.

[16] W. Xu, C. Cheng, X. Song, and P. Zhang, "Experimental investigation of cryogenic flow quenching of horizontal stainless steel tubes," Cryogenics (Guildf)., vol. 117, p. 103327, 2021, doi: https://doi.org/10.1016/j.cryogenics.2021.103327.

[17] Y. Liu et al., "The Effect of High Frequency Pulse on the Discharge Probability in Micro EDM," IOP Conf. Ser. Mater. Sci. Eng., vol. 281, no. 1, pp. 0-7, 2017, doi: 10.1088/1757899X/281/1/012031.

[18] S. Abulais, "Current Research trends in Electric Discharge Machining(EDM):Review,” vol. 5, no. 6, pp. 100-118, 2014.

[19] K.-C. Fan, J. Torng, W. Jywe, R.-C. Chou, and J.-K. Ye, “3-D measurement and evaluation of surface texture produced by scraping process," Measurement, vol. 45, no. 3, pp. 384-392, 2012, doi: https://doi.org/10.1016/j.measurement.2011.11.006.

[20] A. Azam et al., "Design, fabrication, modelling and analyses of a movable speed bump-based mechanical energy harvester (MEH) for application on road," Energy, vol. 214, p. 118894 , 2021, doi: https://doi.org/10.1016/j.energy.2020.118894.

[21] M. D. Fenre and A. Klein-Paste, "Bicycle rolling resistance under winter conditions," Cold Reg. Sci. Technol., vol. 187, p. 103282, 2021, doi: https://doi.org/10.1016/j.coldregions.2021.103282.

[22] S. Sauer and M. Clatworthy, "Editorial Commentary: The Ratio of Tibial Slope and Meniscal Bone Angle is a Strong Predictor for Anterior Cruciate Ligament Injury: A Steep Hill and a Shallow Speed Bump are a Hazardous Combination," Arthrosc. J. Arthrosc. Relat. Surg., vol. 37, no. 5, pp. 1610-1611, 2021, doi: https://doi.org/10.1016/j.arthro.2021.02.006.

[23] G. Monrrabal, A. Bautista, S. Guzman, C. Gutierrez, and F. Velasco, "Influence of the cold working induced martensite on the electrochemical behavior of AISI 304 stainless steel surfaces," J. Mater. Res. Technol., vol. 8, no. 1, pp. 1335-1346, 2019, doi: https://doi.org/10.1016/j.jmrt.2018.10.004.

[24] K. Sritharan, V. Manikandan, and K. Srinivasan, "Influence of solute-solvent interactions at the crystal-solution interface on the growth and morphology of the nonlinear optical single 
crystal methyl-p-hydroxybenzoate (p-MHB)," J. Mol. Struct., vol. 1243, p. 130870, 2021, doi:

https://doi.org/10.1016/j.molstruc.2021.130870.

[25] D. Valdés, S. Martinková, J. Málek, and J. Barták, “Crystal growth in Ge-Sb-Se glass and its relation to viscosity and surface diffusion," J. Non. Cryst. Solids, vol. 566, p. 120865, 2021, doi: https://doi.org/10.1016/j.jnoncrysol.2021.120865.

[26] J.-C. Ren, D. Liu, and Y. Wan, "Modeling and application of Czochralski silicon single crystal growth process using hybrid model of data-driven and mechanism-based methodologies," $J$. Process Control, vol. 104, pp. 74-85, 2021, doi: https://doi.org/10.1016/j.jprocont.2021.06.002.

[27] J.-S. Zhou et al., "Crystal growth of clinopyroxene in mafic alkaline magmas," Earth Planet. Sci. Lett., vol. 568, p. 117005, 2021, doi: https://doi.org/10.1016/j.eps1.2021.117005.
S. Martinková, D. Valdés, S. Slang, K. Pálka, and J. Barták, "Relationship between crystal growth and surface/volume mobilities in Se95Te5 bulk glasses and thin films," Acta Mater., vol. 213, p. 116953, 2021, doi:

https://doi.org/10.1016/j.actamat.2021.116953.

[29] H. Yang and H. Xue, "Effect of cold working on crack growth rate of environmentally assisted cracking of $316 \mathrm{~L} \mathrm{SS}$,"

Structures, vol. 28, pp. 446-455, 2020, doi: https://doi.org/10.1016/j.istruc.2020.09.006.

[30] B. Yang, M. Wu, X. Li, J. Zhang, and H. Wang, "Effects of cold working and corrosion on fatigue properties and fracture behaviors of precipitate strengthened Cu-Ni-Si alloy," Int. J. Fatigue, vol. 116, pp. 118-127, 2018, doi: https://doi.org/10.1016/j.ijfatigue.2018.06.017. 